\title{
POLAR COORDINATES INDUCED BY ACTIONS OF COMPACT LIE GROUPS
}

\author{
BY \\ JIRI DADOK ${ }^{1}$
}

\begin{abstract}
Let $G$ be a connected Lie subgroup of the real orthogonal group $O(n)$. For the action of $G$ on $\mathbf{R}^{\prime \prime}$, we construct linear subspaces a that intersect all orbits. We determine for which $G$ there exists such an a meeting all the $G$-orbits orthogonally; groups that act transitively on spheres are obvious examples. With few exceptions all possible $G$ arise as the isotropy subgroups of Riemannian symmetric spaces.
\end{abstract}

Introduction. Let $G$ be a compact Lie group acting on a real vectorspace $V$, and let $\langle\cdot, \cdot\rangle$ be a $G$-invariant inner product on $V$. Having a linear cross-section a $\subset V$ of minimal possible dimension $\left(\operatorname{dim} a=\min _{x \in V} \operatorname{codim}\{G \cdot x\}\right)$ can often be used to an advantage: In studying $G$-invariant differential equations it can be used for "reduction of variables" (see e.g. [4]); another obvious use is in analyzing the $G$-orbit structure of $V$. We show (Lemma 1) that such cross-sections always exist and how to construct them. In fact, this is extremely simple, the idea is to exploit the critical points of the function $g \rightarrow\langle g \cdot v, w\rangle$, as was done by Hunt [6] to prove the conjugacy of Cartan subalgebras.

The nicest situation arises when the $G$-orbits are orthogonal to the cross-section $a$. It is then natural to think of $a$ and the $G$-orbits as giving polar coordinates on $V$, in analogy with the standard action of $S O(n)$ on $\mathbf{R}^{n}$. Therefore we call real representations of $G$, whose orbits admit orthogonal linear cross-sections, polar.

If $H / G$ is a symmetric space, then the action (here called symmetric space action) of $G$ on the tangent space $T_{e G}$ to $H / G$ at $e G$ is polar [3]. It turns out that if $\pi$ : $G_{1} \rightarrow O(V)$ is any polar representation, then there is a symmetric space $H / G$ and a real isometry $A: V \rightarrow T_{e G}$ mapping $G_{1}$-orbits in one-to-one fashion onto $G$-orbits (Proposition 6). Therefore all polar coordinates on real vectorspaces induced by actions of compact Lie groups can be obtained from the symmetric space actions. In fact there are very few polar actions that are not symmetric space actions, so the methods for determining polar representations could be used to classify symmetric spaces.

Theorems 9 and 10 give the classification of all real irreducible polar representations. Theorem 4 states to what extent these determine all polar representations.

Received by the editors December 1, 1983.

1980 Mathematics Subject Classification. Primary 22E45.

Key words and phrases. Orbits, linear cross sections.

${ }^{1}$ Supported by NSF Grant MCS 81-01635. 
For actions of complex linear reductive groups $G_{\mathbf{C}}$ on a complex vectorspace one cannot hope to find a linear transversal cross-section of all the orbits (recall the Jordan canonical form). However one may hope to find such a cross-section for the closed $G_{\mathrm{C}}$-orbits. This is investigated in [1].

In $\S 1$ we state our results in detail and provide some examples and discussion. The proofs of the results appear in $\S 2$.

1. Polar representations. Let $G$ be a compact Lie group and $g$ its Lie algebra. We shall consider representations $\pi: G \rightarrow O(V)$ on a real vectorspace $V$ preserving an inner product $\langle\cdot, \cdot\rangle$. To construct linear cross-sections of $G$-orbits we shall use

Lemma 1. For $v \in V$ let $\mathfrak{a}_{v} \equiv\{u \in V \mid\langle u, \mathfrak{g} \cdot v\rangle=0\} \equiv(\mathfrak{g} \cdot v)^{\perp}$. The linear space $a_{0}$, meets every $G$-orbit.

The space $\mathrm{g} \cdot v$ is the tangent space to the $G$-orbit through $v$. To obtain a cross-section of minimal dimension we should clearly choose $v$ on a maximal dimensional orbit. In general the minimal cross-sections may contain orbits of maximal dimension and therefore they depend very much on the choice of $v$. For example, if $G=S O(2)$ acts by left multiplication on $V \equiv 2 \times 2$ real matrices, then choosing $v=\left(\begin{array}{ll}1 & 0 \\ 0 & 1\end{array}\right)$ yields $V=S O(2) \cdot \mathfrak{a}_{v}, \mathfrak{a}_{v} \equiv$ symmetric matrices, whereas choosing $u=\left(\begin{array}{ll}1 & 0 \\ 0 & 0\end{array}\right)$ yields $V=S O(2) \cdot a_{u}, a_{u} \equiv$ upper triangular matrices. We are interested in representations for which the minimal dimensional cross-sections are all $G$-conjugate.

Definition. $v \in V$ is called regular if $\mathfrak{g} \cdot v$ is of maximal possible dimension.

Proposition 2. Fix $v_{0} \in V$ a regular element. The following are equivalent:

(i) For any $v \in V$ regular, $\mathfrak{g} \cdot v=k \cdot\left(\mathfrak{g} \cdot v_{0}\right)$ for some $k \in G$.

(ii) For any $v \in V$ regular, $a_{{ }}=k \cdot a_{v_{0}}$ for some $k \in G$.

(iii) For any $u \in a_{v_{0}},\left\langle\mathfrak{g} \cdot u, a_{v_{0}}\right\rangle=0$.

Thus we have uniqueness ( up to the action of $G$ ) of minimal cross-sections if and only if the orbits intersect one such cross-section orthogonally.

Definition. A representation $\pi: G \rightarrow O(V)$ with properties of Proposition 2 is called polar. Any minimal cross-section $a_{v}$, is called a Cartan subspace. The fundamental property of polar representations (allowing induction arguments) is the following inheritability result.

TheOREM 3. Let $\pi: G \rightarrow O(V)$ be any representation of a compact $G$. Fix $w \in V$ and let $G_{n}$ and $\mathfrak{g}_{n}$ be the isotropy subgroup at $w$ and its Lie algebra. Let $\tilde{\pi}$ : $G_{n} \rightarrow O\left(a_{n}\right)$ be given by restriction. Then $\pi$ is polar if and only if $\tilde{\pi}$ is polar and $\langle\mathfrak{a} \cdot \mathfrak{a}, \mathfrak{a}\rangle=0$ for a Cartan subspace $a$ of the $G_{n}$ action on $\mathfrak{a}_{n}$.

Before classifying the irreducible polar representations, we shall investigate to what extent they determine all polar representations. As an example consider $G=\operatorname{Spin}(7)$ and $V=\mathbf{R}^{7} \oplus \mathbf{R}^{8}$ with the standard $(S O(7))$ action on the first factor and the spin representation on the second. This is a polar reducible representation with $G$ being a 
simple Lie group, however the $G$-orbits coincide with $G_{2} \times S O(6)$ orbits: $G_{2}$ acts on $\mathbf{R}^{7}$ by the unique 7-dimensional representation and $S O(6) \simeq S U(4) / \mathbf{Z}_{2}$ acts on $\mathbf{R}^{8} \simeq \mathbf{C}^{4}$ by the standard representation of $S U(4)$. This is an example of

THEOREM 4. Let $\pi: G \rightarrow O(V)$ be a polar representation of the connected compact Lie group $G$. Assume that $V=V_{1} \oplus V_{2}$ is a $G$ stable decomposition. Then:

(i) $\pi: G \rightarrow S O\left(V_{i}\right), i=1,2$, are polar representations. Every Cartan subspace a of $V$ is of the form $a=a_{1} \oplus a_{2}$ with $a_{i}$ being Cartan subspaces of $V_{i}$.

(ii) Fix a Cartan subspace $a=a_{1} \oplus a_{2}$. Let $h_{1}=Z\left(a_{2}\right)$ (the centralizer of $a_{2}$ in $g$ ) and $h_{2}=Z\left(a_{1}\right)$. Further, let $H_{i}$ be the connected Lie subgroups corresponding to $h_{i}$ and define $\rho: H_{1} \times H_{2} \rightarrow S O\left(V_{1} \oplus V_{2}\right)$ by

$$
\rho\left(h_{1}, h_{2}\right)\left(v_{1}, v_{2}\right)=\left(\pi\left(h_{1}\right) v_{1}, \pi\left(h_{2}\right) v_{2}\right) .
$$

Then $\rho$ is a polar representation and the orbits of $\rho$ coincide with the orbits of $\pi$.

Part (ii) of Theorem 4 shows that as far as orbit classification determination of the invariants and Weyl groups (see below), understanding the irreducible representations is sufficient.

Next we shall examine how many times an orbit intersects a Cartan subspace. We let $W=N_{G}(\mathfrak{a}) / Z_{G}(\mathfrak{a})$. The Lie algebra of the normalizer is also the Lie algebra of the centralizer and thus $W$ is a finite group.

THEOREM 5. Let $\pi: G \rightarrow O(V)$ be a polar representation, with a $\subset V$ a Cartan subspace. Then the intersection of $a G$-orbit with a is a single $W$-orbit.

Definition. For $G$ connected we call $\pi: G \rightarrow S O(V)$ a symmetric space representation if there is a real semisimple Lie algebra $h$ with a Cartan decomposition $h=k \oplus \not$, and if there is a Lie algebra isomorphism $A: \mathfrak{g} \rightarrow k$ and a $\mathbf{R}$-vectorspace isomorphism $L: V \rightarrow \not p$ such that $L \circ \pi(X)(y)=[A(X), y]$ for all $X \in \mathfrak{a}, y \in \not$.

Symmetric space representations are of course polar, here the Cartan subspace is a maximal abelian subalgebra of $\not$. Below we shall characterize these representations among the polar representations. It will follow that:

Proposition 6. Let $\pi: G \rightarrow S O(V)$ be a polar representation of a connected Lie group $G$. Then there exists a connected Lie group $\tilde{G}$ with symmetric space representation $\tilde{\pi}: \tilde{G} \rightarrow S O(V)$ such that the $G$-and $\tilde{G}$-orbits in $V$ coincide.

From the above proposition it follows that $W$ is always a reflection group and that the invariants for a polar representation and their properties follow from the theory of symmetric spaces.

Now we turn to the classification of irreducible real polar representations of connected, compact Lie groups $G$. Let us first recall what are the real irreducible representations of such a group $G$. Let $\mathfrak{g}_{\mathbf{C}}$ and $V_{\mathbf{C}}$ be complexifications of $\mathfrak{g}$ and $V$. The representations that stay irreducible when complexified are called orthogonal. If $\pi: \mathfrak{g}_{\mathbf{C}} \rightarrow \operatorname{End}\left(V_{\mathbf{C}}\right)$ is reducible, then in fact $V$ itself has a complex structure and $\pi:$ $\mathrm{g}_{\mathbf{C}} \rightarrow \operatorname{End}(V)$ is irreducible. In this case if $\pi$ (as a complex representation on $V$ ) is self-contragredient it is called symplectic, otherwise it is called unitary. Let $G$ now be 
semisimple. Its complex representations are determined by the theorem of highest weight. Let $\Sigma$ be the set of all roots of $a_{\mathbf{C}},\left\{\alpha_{1} \cdots \alpha_{k}\right\} \equiv \Delta$ a basis for $\Sigma$ and $\lambda_{1} \cdots \lambda_{k}$ the fundamental weights $2\left(\lambda_{i}, \alpha_{j}\right) /\left(\alpha_{j}, \alpha_{j}\right)=\delta_{i j}$, where $($,$) is the inner$ product defined by the Killing form. The theorem of highest weight associates to each $\lambda=\sum_{i=1}^{k} n_{i} \lambda_{i}, n_{i} \in \mathbf{Z}^{+}$, an irreducible complex representation $\pi_{\lambda}: G \rightarrow$ $\operatorname{Aut}\left(V_{\lambda}\right)$. We need to decide for which $\lambda$ is $\pi_{\lambda}$ orthogonal, unitary, or symplectic.

Proposition 7. There is a subset $\mathcal{O}=\left\{\beta_{1} \cdots \beta_{l}\right\} \subset \Sigma^{+}$of strongly orthogonal roots such that

(i) $s_{0}=s_{\beta_{1}} \cdot s_{\beta_{2}} \cdots s_{\beta_{l}}$ is the Weyl group element that maps the positive Weyl chamber into its negative,

(ii) $\pi_{\lambda}$ is unitary if and only if $\lambda \notin \operatorname{span}_{\mathbf{R}}\left\{\beta_{1} \cdots \beta_{l}\right\}$,

(iii) $\pi_{\lambda}$ is symplectic (resp. orthogonal) if and only if $\lambda \in \operatorname{span}_{\mathbf{R}}\left\{\beta_{1} \cdots \beta_{l}\right\}$ and

$$
k(\lambda) \equiv \sum_{i=1}^{l} \frac{\left(\beta_{i}, \lambda\right)-\left(\beta_{i}, s_{0} \lambda\right)}{\left(\beta_{i}, \beta_{i}\right)}
$$

is an odd (resp. even) integer.

If $g_{\mathbf{C}}$ is a simple Lie algebra, then the set $\left\{\beta_{1} \cdots \beta_{l}\right\}$ is chosen as follows: $\beta_{1}$ is the highest root. The root system $\left\{\alpha \in \Sigma \mid\left(\alpha, \beta_{1}\right)=0\right\}$ is either irreducible or equals $\left\{\alpha_{2},-\alpha_{2}\right\} \cup \Sigma_{1}$, with $\Sigma_{1}$ being irreducible and $\alpha_{2} \in \Sigma^{+}$. In the latter case set $\beta_{2}=\alpha_{2}$. The irreducible system that is left inherits its ordering form $\Sigma$ and we proceed by induction, $\beta_{3}$ being the highest root of $\Sigma_{1}$, etc. For semisimple $g_{C}$ we take the union of roots chosen in each simple factor (order is not important). For simple $\mathrm{g}_{\mathbf{C}}$ we list the integers $k(\lambda)$ in the appendix.

REMARK. Let $U^{k}\left(g_{C}\right)$ be the $k$ th level in the natural filtration of the universal enveloping algebra of $\mathfrak{g}_{\mathbf{C}}=U\left(\mathfrak{g}_{\mathbf{C}}\right)$. Fix $\pi_{\lambda}: \mathfrak{g}_{\mathbf{C}} \rightarrow \operatorname{End}\left(V_{\lambda}\right)$ as a representation with a highest weight $\lambda$, and fix $v_{\lambda} \in V_{\lambda}$ as a nonzero highest weight vector. It is not hard to check that $s_{0} v_{\lambda} \in U^{k(\lambda)}\left(\mathrm{g}_{\mathrm{C}}\right) \cdot v_{\lambda}$ but $s_{0} v_{\lambda} \notin U^{\prime}\left(\mathrm{g}_{\mathrm{C}}\right) \cdot v_{\lambda}$ for any $l<k(\lambda)$.

We shall now classify the orthogonal polar representations. Every compact connected Lie group $G$ is covered by $T^{n} \times G_{s}$, where $T^{n}$ is the compact $n$ torus and $G_{s}$ is a semisimple, simply connected Lie group. If $\pi: T^{n} \times G_{s} \rightarrow S O(V)$ is an irreducible, orthogonal representation, then $\left.\pi\right|_{T^{n}}$ is trivial. We shall therefore assume that $G=G_{s}$ and $V=V_{\lambda}^{\mathbf{R}}$ is a real $G$-invariant subspace of the representation space $V_{\lambda}$ for the representation $\pi_{\lambda}: \mathfrak{g}_{\mathbf{C}} \rightarrow V_{\lambda}$ of highest weight $\lambda$.

Definition. A highest weight of a simple Lie algebra $g_{C}$ is called symmetric if all nonzero $\langle\lambda, \alpha\rangle, \alpha \in \Sigma^{+}$are equal. A highest weight of a semisimple $g_{\mathbf{C}}$ is called symmetric if it is symmetric for each of its simple factors.

Proposition 8. If $\pi_{\lambda}: G \rightarrow S O\left(V_{\lambda}^{\mathbf{R}}\right)$ is polar, then $k(\lambda)=2$.

Note that this proposition implies that $G$ can have at most four simple factors on which the representation is nontrivial.

THEOREM 9. (i) If $k(\lambda)=2$, then $\pi_{\lambda}: G \rightarrow S O\left(V_{\lambda}^{\mathbf{R}}\right)$ is always polar. The only such $\pi_{\lambda}$ that are not symmetric space representations are the 7-dimensional representation of 
$G_{2}$ and the spin representation of Spin(7). Both of these representations are transitive on the spheres of dimensions 6 and 7 respectively.

(ii) If $k(\lambda)=4$, then $\pi_{\lambda}: G \rightarrow S O\left(V_{\lambda}^{\mathbf{R}}\right)$ is a symmetric space representation (and therefore polar) iff $\lambda$ is symmetric. The only polar representation with $k(\lambda)=4$ that is not a symmetric space representation is the action of $\operatorname{Spin}(7) \times S U(2)$ on $\mathbf{R}^{8} \otimes \mathbf{R}^{3}$ by the spin $\otimes$ adjoint representation.

The list of all irreducible polar representations is provided in $\$ 2$.

Next assume that $\pi: G \rightarrow S O(V)$ is irreducible but not orthogonal, so $V$ has a $G$-invariant complex structure. If $G$ is covered by $T^{n} \times G_{s}, G_{s}$ semisimple simply connected, then the irreducibility of $\pi$ forces $\left.\pi\right|_{T^{n}}$ to be a character, and hence has a $(n-1)$-dimensional kernel. Therefore we shall assume from now that $G=T^{1} \times G_{s}$. Let $\pi_{\lambda}: G_{s} \rightarrow$ Aut $V_{\lambda}$ be the (complex) representation of $G_{s}$ of highest weight $\lambda$. Let $\pi_{\lambda}^{\theta}$ be the representation $\pi_{\lambda}^{\theta}=e^{i \theta} \otimes \pi_{\lambda}$ of $T^{1} \times G_{s}$ on $\mathbf{C} \otimes V_{\lambda} \simeq V_{\lambda}$ (here $T^{1}$ acts on $\mathrm{C}$ by “multiplication by $e^{i \theta}$ "). Considered as a real representation, $\pi_{\lambda}^{\theta}$ is irreducible.

REMARK. Taking other nonzero characters on $T^{1}$ will not change the orbit structure. If $T^{1}$ is allowed to act trivially, then $\pi_{\lambda}$ as a real representation may be polar (only if $\pi_{\lambda}^{\theta}$ is polar, not conversely). For example $S U(n)$ on $\mathbf{C}^{n} \simeq \mathbf{R}^{2 n}$ is such a representation. We shall classify only the polar representations $\pi_{\lambda}^{\theta}$; in each case it is trivial to check if the $T^{1}$ action is essential or not. It is essential for example in the $T^{1} \times S O(n) \simeq S O(2) \times S O(n)$ action on $\mathbf{R}^{2} \otimes \mathbf{R}^{n} \simeq \mathbf{C}^{n}$.

THEOREM 10. Let $\pi_{\lambda}^{\theta}: T^{1} \times G_{s} \rightarrow S O\left(V_{\lambda}\right)$ be as above. Then:

(i) If $\pi_{\lambda}^{\theta}$ is polar, then $k(\lambda)=1$ or 2 .

(ii) $\pi_{\lambda}^{\theta}$ is a symmetric space representation if and only if $k(\lambda)=1$ or 2 and $\lambda$ is symmetric. (The list is provided in $\$ 2$.)

(iii) If $\pi_{\lambda}^{\theta}$ is polar and $\lambda$ is not symmetric, then $G_{s}=\operatorname{Sp}(n), n>1, G_{2}$ or $\operatorname{Spin}(7)$ and $\pi_{\lambda}$ is the standard, the 7-dimensional and the spin representation, respectively.

\section{The proofs.}

Proof of Lemma 1. Fix $w \in V$ and consider the function $g \rightarrow\langle g \cdot w, v\rangle$. Let $g_{0} \in G$ be a critical point of this function (e.g. a maximum; $G$ is coinpact). The critical point equations say that $0=\left\langle\mathfrak{g} \cdot g_{0} \cdot w, v\right\rangle=\left\langle g_{0} \cdot w, \mathfrak{g} \cdot v\right\rangle$. Thus $g_{0} \cdot w$ $\in a_{v}$.

Proof of Proposition 2. Clearly (i) $\Leftrightarrow$ (ii).

Next we show (i) $\Rightarrow$ (iii). Let $b=\left\{v \in a_{v_{0}}\left\langle g g \cdot v, a_{v_{0}}\right\rangle=0\right\}$.

The set $b$ is a subspace of $a_{v_{0}}$. If it is not the entire $a_{v_{0}}$ we see that $G \cdot b \subset V$ is a closed subset of lower (Hausdorf) dimension that $\operatorname{dim} V$. Consequently there is a regular $w \in V$ and $w \notin G \cdot b$. In fact since the entire $G \cdot w$ misses $G \cdot b$ we may take $w \in \mathfrak{a}_{v_{0}}$. By assumption, for some $k \in G, k \cdot \mathfrak{a}_{w}=\mathfrak{a}_{v_{0}}$ but that means that

$$
(g \cdot k \cdot w)^{\perp}=a_{v_{0}}
$$

or $w \in b$, giving a contradiction. 
Finally (iii) $\Rightarrow$ (i). Let $w \in V$ be regular and let $k \in G$ be such that $k \cdot w \in a_{{ }_{0,0}}$. Because $G$ preserves the inner product we have $k \cdot \mathfrak{a}_{w^{\prime}}=(\mathfrak{g} \cdot k \cdot w)^{\perp}$. By assumption then $k \cdot \mathfrak{a}_{n^{\prime}}=\mathfrak{a}_{\prime^{\prime} 0}$.

Proof of Theorem 3. Assume $\pi: G \rightarrow O(V)$ is polar. If $w \in V$ choose a Cartan subspace such that $w \in \mathfrak{a}$. Since $\mathfrak{g} \cdot w \subset \mathfrak{a}^{\perp}$ we have $\mathfrak{a}_{w} \supset \mathfrak{a}$. Let $v \in \mathfrak{a}$ be regular. We claim that

$$
V=\mathfrak{a} \oplus \mathfrak{g}_{\mathfrak{u}^{\prime}} \cdot v \oplus \mathfrak{g} \cdot \mathfrak{w}, \quad \text { orthogonal sum. }
$$

The sum is orthogonal since $\langle\mathfrak{g} \cdot \mathfrak{a}, \mathfrak{a}\rangle=0$, and since

$$
\left\langle\mathfrak{g}_{\mathfrak{k}} \cdot v, \mathfrak{g} \cdot w\right\rangle=-\left\langle v, \mathfrak{g} \cdot\left(\mathfrak{g}_{\mathfrak{w}^{*}} \cdot w\right)\right\rangle-\left\langle v,\left[\mathfrak{g}_{n^{\prime}}, \mathfrak{g}\right] \cdot w\right\rangle=0 .
$$

The sum is equal to $V$ by a dimension count, since we already know that $V=a \oplus a$ $\cdot v$. Therefore $\mathfrak{a}_{w}=\mathfrak{a} \oplus \mathfrak{g}_{n} \cdot v=\mathfrak{a} \oplus \mathfrak{g}_{w} \cdot a$ and the representation of $G_{w}$ on $\mathfrak{a}_{n}$ is polar. Its Cartan subspaces are also Cartan subspaces for the $G$ action on $V$.

Conversely assume that $\mathfrak{a}_{n}=\mathfrak{a} \oplus \mathfrak{g}_{w^{*}} \cdot \mathfrak{a}$ and that $\langle\mathfrak{g} \cdot \mathfrak{a}, \mathfrak{a}\rangle=0$. By Lemma 1 all $G$-orbits meet $\mathfrak{a}_{n}$, and by assumption all $G_{w}$-orbits in $\mathfrak{a}_{n}$. meet a. Hence all $G$-orbits meet $a$. Let $v \in a$ be regular. We see that $a_{1}, \supset a$. On the other hand if $a$ intersects all orbits it cannot be of codimension larger than the maximal dimension of an orbit. We therefore have $a_{1}=a$ and the proof of the theorem is complete.

Proof of Theorem 4. Let $V=V_{1} \oplus V_{2}$ and $a \subset V$ be a Cartan subspace. If $v \in \mathfrak{a}$ is regular let $v=v_{1}+v_{2}$ with $v_{i} \in V_{i}$. Since $V_{i}$ are $G$ stable it is clear from $V=\mathfrak{a} \oplus \mathfrak{g} \cdot v$ that

$$
V_{i}=\mathfrak{a}_{i} \oplus \mathfrak{g} \cdot v_{i} \quad \text { and } \quad \mathfrak{a}_{1} \oplus \mathfrak{a}_{2}=\mathfrak{a} .
$$

Thus the representation of $G$ on $V_{i}, i=1,2$, is polar.

Next let $a_{1} \subset V$ be any Cartan subspace for the polar action of $G$ on $V_{1}$. Fix $v_{1} \in \mathfrak{a}_{1}$ a regular element for this action and set $h_{2} \equiv \mathfrak{a}_{v_{1}}=Z_{\mathfrak{g}}\left(\mathfrak{a}_{1}\right)$ (the last equality follows from Theorem 3). Considering the action of $G$ on $V$ we observe that $a_{n_{1}}=a_{1} \oplus V_{2}$. Theorem 3 now implies that the Cartan subspaces for the action of $G$ on $V_{2}$ are the same as for the action of $G_{v^{\prime}}$ on $V_{2}$ and that if $a_{2} \subset V_{2}$ is any such Cartan subspace, then $a=a_{1} \oplus a_{2}$ is a Cartan subspace for the $G$ action on $V$. This proves (i).

Fix now also $\mathfrak{a}_{2} \subset V$ a Cartan subspace and a regular element $\left(v_{1}+v_{2}\right) \in\left(\mathfrak{a}_{1}+\right.$ $\left.\mathfrak{a}_{2}\right)=a$ for the $G$ action on $V$. Let $h_{1}=\mathfrak{g}_{v_{2}} \equiv Z_{\mathfrak{a}}\left(\mathfrak{a}_{2}\right)$. From the proof of part (i) we have that $h_{2} \cdot v_{1}=\mathfrak{g} v_{1}$. This implies that $\mathfrak{g}=h_{1}+h_{2}$ and that $G=H_{1} \cdot H_{2}$, where the $H_{i}$ are the connected components of $G_{v^{\prime}}$ (here we used the connectedness of $G$ ). We now consider the $H_{1} \times H_{2}$ action on $V_{1} \oplus V_{2}\left(H_{i}\right.$ acts on $V_{i}$ only). If $\left(u_{1}+u_{2}\right)$ $\in\left(a_{1}+a_{2}\right)$ we see (from $\left.G=H_{1} \cdot H_{2}=H_{2} \cdot H_{1}\right)$ that $G \cdot\left(u_{1}+u_{2}\right) \subset H_{1} \times H_{2}$. $\left(u_{1}+u_{2}\right)$, and from $\mathrm{g}=h_{1}+h_{2}$ we conclude that the above orbits are of the same dimension. By connectedness they are equal.

Proof of Theorem 5. Suppose $u, v \in a$ are on the same $G$-orbit, so $g \cdot u=v$ for some $g \in G$. Both $a$ and $g \cdot a$ are Cartan subspaces containing $v$ and therefore (by Theorem 3) there is $h \in G_{v}$, such that $h \cdot(g \cdot \mathfrak{a})=\mathfrak{a}$. Thus $h g$ represents an element in $W$ and we have $h g \cdot u=h \cdot v=v$. 
Proof of Proposition 7. It is enough to give a proof for $G$ simple. Part (i) may be verified on a case by case basis which is easier than an abstract argument. It can also be seen that $s_{0} \equiv-I$ on $\operatorname{span}_{\mathbf{R}}\left\{\beta_{1} \cdots \beta_{l}\right\}$, and $s_{0}=+I$ on the orthocomplement. From this follows part (ii) since $\pi_{\lambda}$ is self-contragredient if and only if $s_{0} \lambda=-\lambda$. To prove part (iii) let $u \subset g_{C}$ be the subalgebra generated by the rootspaces of $g_{C}$ corresponding to $\pm \beta_{1} \cdots \pm \beta_{l}$. Now $u$ is isomorphic to a direct sum of $l$ copies of $\operatorname{sl}(2, \mathbf{C})$. We restrict $\pi_{\lambda}$ to $u$ and let $U_{\lambda} \in V_{\lambda}$ be the unique irreducible $u$-module generated by the $\lambda$-weight space of $\pi_{\lambda}$. Here we used that $\lambda \in \operatorname{span}_{\mathbf{R}}\left\{\beta_{1} \cdots \beta_{l}\right\}$. If $B: V_{\lambda} \times V_{\lambda} \rightarrow \mathbf{C}$ is the nondegenerate bilinear form invariant under $\pi_{\lambda}$, we see that $B$ must remain nondegenerate on $U_{\lambda} \times U_{\lambda} \stackrel{B}{\rightarrow} \mathbf{C}$ since the $u$-module $U_{\lambda}$ appears in $V_{\lambda}$ with multiplicity one. Recall that $\pi_{\lambda}$ is orthogonal (resp. symplectic) according to whether $B$ is symmetric (resp. skew symmetric). This now can be decided on the $u$-module $U_{\lambda}$. The proposition now follows from the fact that for $s l(2, \mathrm{C})$ the irreducible representation $\pi_{\mu}, \mu=k \alpha / 2$ (where $\alpha$ is the positive root of $s l(2, \mathbf{C}))$, is orthogonal if $k$ is even, and is symplectic if $k$ is odd.

Before proving Proposition 8 and Theorem 9 we do some preparatory work. Assume that $\pi_{\lambda}: G \rightarrow$ Aut $V_{\lambda}$ is an orthogonal representation of a semisimple Lie group $G$ with highest weight $\lambda$. Let $\mathscr{A}$ be the set of weights of $\pi_{\lambda}$ and define

$$
\mathscr{A}_{\lambda} \equiv\left\{\mu \in \mathscr{A}: \pm \mu \neq \lambda \text { and } \pm \mu \neq \lambda-\alpha, \alpha \in \Sigma^{+}\right\} \text {. }
$$

LeMma 11. Assume that $k(\lambda) \geqslant 4$ and $\mu, \mu-\alpha \in \mathscr{A}_{\lambda}$ with $(\lambda, \mu) \neq \pm(\lambda, \mu-\alpha)$. Then $\pi_{\lambda}$ is not polar.

Proof. Let $V_{\lambda}^{\mathbf{R}} \subset V_{\lambda}$ be a real invariant subspace and let $p: V_{\lambda} \rightarrow V_{\lambda}^{\mathbf{R}}$ be the orthogonal projection (there is a Hermitian invariant inner product on $V_{\lambda}$ that restricts to a real inner product on $V_{\lambda}^{\mathbf{R}}$ ). If $V_{\lambda}^{\mu}$ is the $\mu$-weight space of $V_{\lambda}$, then

$$
p\left(V_{\lambda}^{\mu}\right)=p\left(V_{\lambda}^{-\mu}\right) \text { and }\left\langle p\left(V_{\lambda}^{\mu}\right), p\left(V_{\lambda}^{\eta}\right)\right\rangle=0 \quad \text { if } \mu \neq \pm \eta \text {. }
$$

Now let $v \in V_{\lambda}$ be a highest weight vector and set $w=p(v)$. Recall that $a_{w}=$ $(\mathrm{g} \cdot w)^{\perp}$. It is clear that $p\left(V_{\lambda}^{\eta}\right) \subset \mathfrak{a}_{w}$ for every $\eta \in \mathscr{A}_{\lambda}$.

Next we claim that if $k(\lambda)=4$, then we cannot have $2 \lambda=\alpha+\beta$ with $\alpha, \beta \in \Sigma^{+}$: First if $\lambda$ is a root, then it is either a dominant short or long root. In either case $k(\lambda)=2$. If $\alpha \neq \beta$, then consider the roots in the 2 -dimensional $\operatorname{span}_{\mathbf{R}}\{\alpha, \beta\}$. In this rank-two root system $\alpha, \beta$ are positive roots and $\lambda$ is a dominant weight. A quick check of rank-two root systems reveals that if $\lambda=(\alpha+\beta) / 2$ is not a root, then $(\alpha, \beta)=0$. Thus the length of $\lambda$ cannot exceed the length of a short root. On the other hand $k(\lambda)=4$ means that $\lambda=\frac{1}{2}\left(\beta_{1}+\beta_{2}+\beta_{3}+\beta_{4}\right), \beta_{i} \in \mathcal{O}$ (not necessarily distinct). But then the length of $\lambda$ is larger than the length of a short root.

Since $2 \lambda$ is not a sum of two roots the complexification of the isotropy subalgebra $\mathfrak{g}_{w}$ equals

$$
\left(g_{w}\right)_{\mathbf{C}}=h_{\lambda} \oplus \underset{\substack{\alpha \in \Sigma \\(\alpha, \lambda)=0}}{\bigoplus} g^{\alpha}, \quad \text { where } h_{\lambda}=\{x \in h: \lambda(x)=0\}
$$


Assume now that $\mu, \mu-\alpha \in \mathscr{A}_{\lambda}$, fix $z \in V_{\lambda}^{\mu}$ such that $X_{-\alpha} \cdot z \neq 0, X_{-\alpha} \in \mathbb{a g}^{-\alpha}$, let $x=p(z)$ and $y=p\left(X_{-\alpha} \cdot z\right)$. Observe that $y \neq 0$ (projection $p$ restricted to a weight space has trivial kernel) and that

$$
y \notin(\mathfrak{g} \cdot x)^{\perp} .
$$

Otherwise

$$
0=\left\langle p\left(X_{-\alpha} \cdot z\right), \mathfrak{g} \cdot p(z)\right\rangle=\left\langle p\left(X_{-\alpha} \cdot z\right), p(\mathfrak{g} \cdot z)\right\rangle=\left\langle p\left(X_{-\alpha} \cdot z\right), \mathfrak{g}_{\mathbf{C}} \cdot z\right\rangle,
$$

so in particular $0=\left\langle p\left(X_{-\alpha} \cdot z\right), X_{-\alpha} \cdot z\right\rangle=\left\|p\left(X_{-\alpha} \cdot z\right)\right\|^{2}$, contradicting $y=$ $p\left(X_{-\alpha} \cdot z\right) \neq 0$. It now follows from (1), (2) and the assumption that $\langle\lambda, \mu\rangle \neq$ $\pm\left\langle\lambda, \mu^{-} \alpha\right\rangle$ that the real $\mathfrak{g}_{n}$-modules $U_{x} \equiv \mathscr{U}\left(\mathfrak{g}_{n}\right) \cdot x$ and $U_{y} \equiv \mathscr{U}\left(\mathfrak{g}_{n}\right) \cdot y$ are orthogonal to each other, and both are in $a_{n}$. Suppose now that the action of $G$ on $V_{\lambda}^{\mathbf{R}}$ is polar. By Theorem 3 the action of $G_{x^{\prime}}$ on $\mathfrak{A}_{n}$ is polar. Let $\mathfrak{a}_{{ }^{\prime}}=U_{x} \oplus U_{y} \oplus U$ (orthogonal direct sum). By Theorem 4 the actions of $G_{w}$ (rather its connected component) on $U_{x}, U_{y}$, and $U$ are polar and we may find a Cartan subspace a for $a_{w}$. that contains both $x, y \in \mathfrak{a}$. Theorem 3 again implies that $\langle\mathfrak{a} \cdot \mathfrak{a}, \mathfrak{a}\rangle=0$. But that contradicts (3). The proof of the lemma is complete.

Proof of Proposition 8. Let $\pi_{\lambda}: G \rightarrow \operatorname{Aut}\left(V_{\lambda}\right)$ be an orthogonal representation with $k(\lambda) \geqslant 6$. Set $k=k(\lambda)$ and $l=k / 2$. By assumption we have $2 \lambda=\beta_{i_{1}}+\beta_{i_{2}}+$ $\cdots+\beta_{i_{k}}, \beta_{i} \in \mathcal{O}$. So there is a weight $\mu$ of $\pi_{\lambda}$ of the form $\mu=\lambda-\beta_{j_{1}}-\beta_{j_{2}}-$ $\cdots-\beta_{j l}, \beta_{j} \in \mathcal{O}$ and $(\mu, \lambda) \leqslant 0$. For some $\beta \in \mathcal{O}, \mu-\beta$ is also a $\pi_{\lambda}$ weight of $\pi_{\lambda}$. Since no root of $\mathfrak{g}_{\mathbf{C}}$ is a sum of two elements of $\mathcal{O}$ it follows that $\mu, \mu-\beta \in \mathscr{A}_{\lambda}$ and Lemma 11 applies.

Lemma 12. Let $\pi_{\lambda}: G \rightarrow \operatorname{Aut}\left(V_{\lambda}\right)$ be orthogonal with $k(\lambda)=4$. Assume that $2 \lambda=3 \beta_{1}+\beta_{2}\left(\right.$ resp. $\left.2 \lambda=2 \beta_{1}+\beta_{2}+\beta_{3}\right), \quad \beta_{i} \in \mathcal{O}$. If there is a Weyl group element $s \in W$ such that $s \beta_{1}=\beta_{2}$, then $\pi_{\lambda}$ is not polar.

Proof. Suppose $2 \lambda=3 \beta_{1}+\beta_{2}$. Then $\mu=\lambda-\beta_{1}-2 \beta_{2}$ is a weight of $\pi_{\lambda}$ with $\langle\mu, \lambda\rangle=0$. But then $\mu, \mu+\beta_{2} \in \mathscr{A}_{\lambda}$ and Lemma 11 applies. The case $2 \lambda=2 \beta_{1}+$ $\beta_{2}+\beta_{3}$ is similar.

Lemma 13. Let $\pi: G \rightarrow \operatorname{Aut}\left(V_{\lambda}\right)$ be an orthogonal representation with $k(\lambda)=4$. Let $2 \lambda=\beta_{1}+\beta_{2}+\beta_{3}+\beta_{4}, \beta_{i} \in \mathcal{O}$, not necessarily distinct. Assume that $\beta_{i}, i=1,2,3,4$, are long roots (each for some simple factor of $\mathrm{g}_{\mathrm{C}}$ ). Suppose there exists $\alpha \in \Sigma^{+}$whose orthogonal projection on $\operatorname{span}_{\mathbf{R}}\left\{\beta_{1}, \beta_{2}, \beta_{3}, \beta_{4}\right\}$ equals one half of one of the roots, say $\beta_{1} / 2$. Then $\pi_{\lambda}$ is not polar.

Proof. If we cannot scale the inner product on each simple factor of $\mathfrak{g}_{C}$ to make the $\left(\lambda, \beta_{i}\right), i=1,2,3,4$, all equal, then Lemma 12 applies (long roots for a simple Lie algebra are on the same $W$ orbit) and $\pi_{\lambda}$ is not polar. So we will assume the $\left(\lambda, \beta_{i}\right)$ are equal.

Next consider the rank-two subalgebra generated by the rootspaces corresponding to $\alpha$ and $\beta_{1}$. We conclude there is a root $\tilde{\alpha}$ such that $\alpha+\tilde{\alpha}=\beta_{1}$, and $\tilde{\alpha}$ also projects onto $\beta_{1} / 2$. 
Now observe that $\lambda-\beta_{2}-\tilde{\alpha}$ and $\lambda-\beta_{2}-\beta_{1} \equiv \mu$ are weights of $\pi_{\lambda}$. Also, we have $(\mu, \lambda)=0$ and $(\mu+\alpha, \lambda)>0$. But $\mu+\alpha=\lambda-\beta_{2}-\tilde{\alpha}$, so if we show that $\mu$ and $\mu+\alpha$ are both in $\mathscr{A}_{\lambda}$, Lemma 11 will show that $\pi_{\lambda}$ is not polar. We argue by contradiction. Suppose that $\lambda-\beta_{2}-\tilde{\alpha}=\lambda-\gamma, \gamma \in \Sigma^{+}$, i.e. $\gamma=\beta_{2}+\tilde{\alpha}$. Then we have

$$
\|\gamma\|^{2}=\left\|\beta_{2}\right\|^{2}+\|\tilde{\alpha}\|^{2}+2\left(\tilde{\alpha}, \beta_{2}\right)>\left\|\beta_{2}\right\|^{2}
$$

This is however impossible since $\gamma$ and $\beta_{2}$ are in the same simple factor, and $\beta_{2}$ is a long root. Similarly we can show that $\mu=\lambda-\beta_{1}-\beta_{2}=\lambda-\gamma, \gamma \in \Sigma^{+}$, leads to a contradiction.

REMARK. All the roots in $\mathcal{O}$ are long unless $\mathfrak{g}_{\mathbf{C}}$ contains a factor of $G_{2}$ or $S O(4 k-1), k=2,3, \ldots$

Proof of Theorem 9. (i) We first list all self-dual representations $\pi_{\lambda}$ with $k(\lambda)=2$. For simple $g_{C}$ we order the roots and the fundamental weights as in Humphreys [5].

Case I. $G$ simple. Besides all the adjoint representations we find

$\begin{array}{lclc}\text { Group } & \text { Representation } & \text { Description } & \begin{array}{c}\text { Corresponds to } \\ \text { symmetric space }\end{array} \\ B_{l}, l \geqslant 3 & \lambda=\lambda_{1} & \text { Standard } & S O(2 l+1,1) / S O(2 l+1) \\ B_{3} & \lambda=\lambda_{3} & \text { Spin } & \text { none } \\ \text { Spin(9) } & \lambda=\lambda_{4} & \text { Spin } & F_{4} / \operatorname{Spin}(9) \\ C_{l}, l \geqslant 2 & \lambda=\lambda_{2} & \Lambda^{2} \mathbf{C}^{2 l} & S U^{*}(2 n) / \operatorname{Sp}(n) \\ D_{l}, l \geqslant 3 & \lambda=\lambda_{1} & \text { Standard } & S O(2 l, 1) / S O(2 l) \\ D_{4} & \lambda=\lambda_{3}, \lambda_{4} & \text { Spin } & S O(8,1) / S O(8) \\ G_{2} & \lambda=\lambda_{2} & \text { 7-dimensional } & \text { none } \\ F_{4} & \lambda=\lambda_{4} & \text { 26-dimensional } & E_{6} / F_{4}\end{array}$

All the representations with the exception of $C_{l}, l>1$, and $F_{4}$ are transitive on spheres, hence polar. To show that the others are polar observe that the zero weight space is a Cartan subspace.

Case II. $G=G_{1} \times G_{2}, \pi_{\lambda}=\pi_{\mu_{1}} \otimes \pi_{\mu_{2}}$, where $\pi_{\mu_{i}}, i=1,2$, are symplectic representations with $k\left(\mu_{i}\right)=1$. This case includes only $G=\operatorname{Sp}(n) \times \operatorname{Sp}(m)$ acting by the tensor product of standard representations. This is a symmetric space representation corresponding to $\mathrm{Sp}(n, m) / \mathrm{Sp}(n) \times \mathrm{Sp}(m)$.

(ii) We now assume that $\pi_{\lambda}$ is self-dual with $k(\lambda)=4$.

Case I. $2 \lambda=\beta_{1}+\beta_{2}+\beta_{3}+\beta_{4}, \beta_{i} \in \mathcal{O}$ (not necessarily distinct), are all long roots.

From Lemmas 12 and 13 it follows that if $\lambda$ is not symmetric, then $\pi_{\lambda}$ is not polar. We list therefore only $\pi_{\lambda}$ with $\lambda$ symmetric. In each case one may verify that $\pi_{\lambda}$ is polar by induction as follows: Choose $w \in V_{\lambda}^{\mathbf{R}}$ as the proof of Lemma 11 and compute the representation of $a_{w}$ on $a_{w}$, which is quite easy. Then use Theorem 3. 


\begin{tabular}{|c|c|c|}
\hline Group & Highest weight & $\begin{array}{l}\text { Corresponds to } \\
\text { symmetric space }\end{array}$ \\
\hline$A_{1}$ & $4 \lambda_{1}$ & $S L(3, \mathbf{R}) / S O(3)$ \\
\hline$A_{7}$ & $\lambda_{4}$ & $E_{7} / S U(8)$ \\
\hline $\left.\begin{array}{l}B_{l}\left(\operatorname{resp} . D_{l}\right), \\
l \geqslant 1(\text { resp. } l \geqslant 3)\end{array}\right\}$ & $2 \lambda_{1}$ & $\left\{\begin{array}{l}S L(n, \mathbf{R}) / S O(n) \\
n=2 l+1(\text { resp. } 2 l)\end{array}\right.$ \\
\hline$C_{4}$ & $\lambda_{4}$ & $E_{6} / \mathrm{Sp}(4)$ \\
\hline$D_{8}$ & $\lambda_{7}, \lambda_{8}$ & $E_{6} / \operatorname{Spin}(16)$ \\
\hline $\left.\begin{array}{l}B_{l_{1}}+B_{l_{2}}, B_{l_{1}}+D_{m_{1}} \\
D_{m_{1}}+D_{m_{2}}, \\
l_{1}, l_{2} \geqslant 2, m_{1}, m_{2} \geqslant 3\end{array}\right\}$ & $\lambda_{1}+\lambda_{1}$ & $S O(p, q) / S O(p) \times S O(q)$ \\
\hline $\left.\begin{array}{l}B_{l}+D_{4}, l \geqslant 1 \\
D_{m}+D_{4}, m \geqslant 3\end{array}\right\}$ & $\lambda_{1}+\lambda_{3}$ or $\lambda_{1}+\lambda_{4}$ & $S O(p, 8) / S O(p) \times S O(8)$ \\
\hline $\left.\begin{array}{l}A_{1}+B_{l}, l \geqslant 2 \\
A_{1}+D_{l}, l \geqslant 3\end{array}\right\}$ & $2 \lambda_{1}+\lambda_{1}$ & $S O(p, 3) / S O(p) \times S O(3)$ \\
\hline$A_{1}+A_{1}$ & $3 \lambda_{1}+\lambda_{1}$ & $G_{2} / S U(2) \times S U(2)$ \\
\hline$A_{1}+C_{3}$ & $\lambda_{1}+\lambda_{3}$ & $F_{4} / \mathrm{Sp}(3) \times S U(2)$ \\
\hline$A_{1}+D_{6}$ & $\lambda_{1}+\lambda_{5}, \lambda_{1}+\lambda_{6}$ & $E_{7} / \operatorname{Spin}(12) \times S U(2)$ \\
\hline$A_{1}+E_{7}$ & $\lambda_{1}+\lambda_{7}$ & $E_{8} / E_{7} \times S U(2)$ \\
\hline$A_{1}+A_{1}+A_{1}$ & $2 \lambda_{1}+\lambda_{1}+\lambda_{1}$ & $S O(7) / S O(4) \times S O(3)$ \\
\hline$A_{1}+A_{1}+A_{1}+A_{1}$ & $\lambda_{1}+\lambda_{1}+\lambda_{1}+\lambda_{1}$ & $S O(8) / S O(4) \times S O(4)$ \\
\hline
\end{tabular}

Case II. $2 \lambda=\beta_{1}+\beta_{2}+\beta_{3}+\beta_{4}$, and at least one $\beta_{i} \in \mathcal{O}$ is short. In this case $G$ has to have a factor of $G_{2}$ or $\operatorname{Spin}(k), k=7,11,15$. A case by case application of Lemma 11 shows that none of these groups have an orthogonal polar representation $\pi_{\lambda}$ with $\lambda$ as described above. For the same reason $G_{2} \times S U(2)$ on $\mathbf{R}^{21}$ (the 7-dimensional $\otimes$ adjoint) and $\operatorname{Spin}(11) \times S U(2)$ on $\mathbf{R}^{64}$ (spin $\otimes$ the standard) are not polar. Now every $s$-simple compact Lie algebra contains an $S U(2)$ subalgebra. Applying Lemma 11 again it is clear that no higher-dimensional representation of semisimple Lie groups may be tensored with the 7-dimensional (resp. 32-dimensional) representations of $G_{2}$ (resp. Spin(1)) to yield a polar representation.

On the other hand $\operatorname{Spin}(7) \times S U(2)$ on $\mathbf{R}^{24}$ is polar. The easiest way to see it is to check that it has a 21-dimensional orbit, which therefore must coincide with the $S O(8) \times S O(3)$ orbit on the same space.

Assume now that there is a polar representation $\pi_{\lambda_{3}} \otimes \pi_{\mu}$ of $\operatorname{Spin}(7) \otimes H$. Then $k(\mu)=2$ and $\pi_{\mu}$ is an orthogonal representation on $V_{\mu}^{\mathbf{R}}$. Fix $x \in \mathbf{R}^{8}$ (representation space for $\operatorname{Spin}(7)$ ) and $y \in V_{\mu}^{\mathbf{R}}$. The isotropy group at $x \otimes y$ is $G_{2} \times H_{v}$. Unless $H=S U(2), y$ may be chosen so that $H_{y}$ has a nontrivial simple factor. By Theorem 3 the representation of $G_{2} \times H_{y}$ on $\mathfrak{A}_{x \otimes y}$ should be polar but we already know (see also Theorem 10) all polar representations involving the 7-dimensional representation of $G_{2}$. We thus have a contradiction. 
We now proceed with the classification of nonorthogonal representations. Thus as discussed in $\S 1$ let $\pi_{\lambda}^{\theta}: G \times T^{1} \rightarrow S O\left(V_{\lambda}\right)$ be the irreducible real representation on the representation space $V_{\lambda}$ viewed as a real vectorspace. The weight spaces of $\pi_{\lambda}$ : $G \rightarrow$ Aut $V_{\lambda}$ thus become real even-dimensional subspaces preserved by $T^{1}$ and the maximal torus in $G$. Define

$$
\mathscr{B}_{\lambda} \equiv\left\{\mu \text { a weight of } \pi_{\lambda}: \mu \neq \lambda-\alpha \text { for all } \alpha \in \Sigma^{+}\right\} .
$$

Corresponding to Lemma 11 we have

LeMmA 14. If for some $\mu \in \mathscr{B}_{\lambda}$ and $\alpha \in \Sigma^{+}, \mu+\alpha$ also belongs to $\mathscr{B}_{\lambda}$, then $\pi_{\lambda}^{\theta}$ is not polar.

Proof. Fix $w \in V_{\lambda}^{\lambda}$. It is clear that

$$
\left(g_{w}\right)_{\mathbf{C}}=h_{\lambda}+\bigoplus_{\substack{\alpha \in \Sigma \\(\alpha, \lambda)=0}} g^{\alpha}, \quad \text { where } h_{\lambda}=\{x \in h: \lambda(x)=0\} .
$$

Assume now that $\pi_{\lambda}^{\theta}$ is polar. Fix $x \in V_{\lambda}^{\mu}$ and set $y \in V_{\lambda}^{\mu+\alpha}$ equal to $y=X_{\alpha} x$, $X_{\alpha} \in \mathrm{g}^{\alpha}$. Let $U_{x}$ and $U_{y}$ be the real $\left(\mathrm{g}+t_{1}\right)_{w}$-modules generated by $x$ and $y$. From (4) it follows that $U_{x}$ and $U_{y}$ are orthogonal to each other $\left(t_{1}\right.$ preserves weight spaces), and by assumption $U_{x} \oplus U_{v} \subset a_{w}$. Since by Theorem 3 the action of $\left(\mathfrak{g}+t_{1}\right)_{w}$ on $\mathfrak{a}_{w}$ is polar we can find a Cartan subspace a (using Theorem 4) containing $x, y \in \mathfrak{a}$. By Theorem 3 again we must have $\langle\mathrm{g} \cdot x, y\rangle=0$, and therefore also $\left\langle g_{\mathbf{C}} \cdot x, y\right\rangle=0$. But that directly contradicts our choice of $x$ and $y$. Thus $\pi_{\lambda}^{\theta}$ is not polar.

Corollary 15. If $\pi_{\lambda}^{\theta}: G \times T^{1} \rightarrow S O\left(V_{\lambda}\right)$ is polar, then $k(\lambda) \leqslant 2$.

Proof. If $\lambda-s_{0} \lambda=\beta_{1}+\beta_{2}+\cdots+\beta_{k}$ with $k \geqslant 3, \beta_{i} \in \mathcal{O}$, then evidently by setting $\mu=s_{0} \lambda$ and $\alpha=\beta_{1}$ Lemma 14 applies. (Recall no root is a sum of two "O-roots".)

Corollary 16. If $\pi_{\lambda}$ is self-dual, $k(\lambda)=2$ and $\pi_{\lambda}^{\theta}$ is polar, then $\mathscr{B}_{\lambda}=\{-\lambda\}$. In particular the orthogonal action of $\pi_{\lambda}: G \rightarrow S O\left(V_{\lambda}^{\mathbf{R}}\right)$ is transitive on the unit sphere.

Proof. The first statement is clear from Lemma 14. From this it follows that if $x \in V_{\lambda}^{\mathbf{R}}$, then $\mathrm{g} \cdot x$ is of codimension one in $V_{\lambda}^{\mathbf{R}}$.

Proof of Theorem 10. We first consider the representation $\pi_{\lambda}$ with $k(\lambda)=1$. $G$ must be necessarily simple and a short computation reveals that only the $A_{l}$ and $C_{l}$ series have such representations; these are the standard representations with $\lambda=\lambda_{1}$ and their duals. Since $T^{1} \times S U(n)\left(\right.$ resp. $\left.T^{1} \times \mathrm{Sp}(n)\right)$ is transitive on the sphere in $\mathbf{R}^{2 n}$ (resp. in $\mathbf{R}^{4 n}$ ) the corresponding representations $\pi_{\lambda_{1}}^{\theta}$ are indeed polar. The representations of $S U(n) \times T^{1}$ are symmetric space representations (corresponding to $S U(n, 1) / S(U(n) \times U(1)))$ whereas the representations of $T^{1} \times \mathrm{Sp}(n)$ are not.

We now consider the representations $\pi_{\lambda}$ with $k(\lambda)=2$. If $G$ is not simple then we must have $G=G_{1} \times G_{2}, \pi_{\lambda}=\pi_{\mu_{1}} \otimes \pi_{\mu_{2}}, G_{i}$ are simple, and $k\left(\mu_{i}\right)=1$. We have just found all the representations with $k(\mu)=1$. A simple application of Lemma 15 shows that if $n, m>1$, then the actions of $\operatorname{Sp}(n) \times \operatorname{Sp}(m)$ and $S U(m) \times \operatorname{Sp}(n)$ by the tensor product of standard representations are not polar: the lowest weight and the lowest weight plus a short root of $\operatorname{Sp}(n)$ are in $\mathscr{B}_{\lambda}$. Thus if $G$ is not simple the only polar $\pi_{\lambda}^{\theta}$ with $k(\lambda)=2$ are the actions of $T^{1} \times S U(n) \times S U(m)$ on $\mathbf{R}^{4 n m}$. 
These are symmetric space representations corresponding to

$$
S U(n, m) / S(U(n) \times U(m)) .
$$

Finally, if $G$ is simple and $k(\lambda)=2$ we list all $\pi_{\lambda}$ that are not self-dual and using the inheritability Theorem 3 we can in each case verify that $\pi_{\lambda}^{\theta}: G \times T^{1} \rightarrow S O\left(V_{\lambda}\right)$ is indeed polar. If $\pi_{\lambda}=\pi_{\lambda}^{*}$ we list only those that are not eliminated by Corollary 16 . Again, all the remaining representations do indeed give polar actions as can be verified case by case using Theorem 3 . We thus obtain the following list:

\begin{tabular}{|c|c|c|}
\hline Group & Highest weight & $\begin{array}{l}\text { Corresponds to } \\
\text { symmetric space }\end{array}$ \\
\hline$A_{l}, l \geqslant 1$ & $2 \lambda_{1}=2 \lambda_{l}^{*}$ & $\operatorname{Sp}(n, \mathbf{R}) / U(n)$ \\
\hline$A_{l}, l \geqslant 3$ & $\lambda_{2}=\lambda_{l-1}^{*}$ & $S O^{*}(2 n) / U(n)$ \\
\hline $\left.\begin{array}{l}B_{l}, l \geqslant 2 \\
D_{l}, l \geqslant 4\end{array}\right\}$ & $\lambda_{1}=\lambda_{1}^{*}$ & $S O(p, 2) / S O(p) \times S O(2)$ \\
\hline$B_{3}$ & $\lambda_{3}=\lambda_{3}^{*}$ & none \\
\hline$D_{4}$ & $\begin{array}{l}\lambda_{3}=\lambda_{3}^{*} \\
\lambda_{4}=\lambda_{4}^{*}\end{array}$ & $S O(8,2) / S O(8) \times S O(2)$ \\
\hline$D_{5}$ & $\lambda_{4}=\lambda_{5}^{*}$ & $E_{6} / S O(10) \times T^{1}$ \\
\hline$G_{2}$ & $\lambda_{2}=\lambda_{2}^{*}$ & none \\
\hline$E_{6}$ & $\lambda_{1}=\lambda_{6}^{*}$ & $E_{7} / E_{6} \times T^{1}$ \\
\hline
\end{tabular}

Appendix. Here we list the integer $k(\lambda)$, where $\lambda$ is a fundamental weight of a simple Lie algebra. The ordering we use is again that of Humphreys [5].

$A_{l}: \quad k\left(\lambda_{j}\right)=k\left(\lambda_{l-j}\right)=j$ for $1 \leqslant j \leqslant(l+1) / 2$.

Also, $\lambda_{j}^{*}=\lambda_{l-j}$.

$B_{l}: \quad k\left(\lambda_{2 j}\right)=k\left(\lambda_{2 j-1}\right)=2 j$ for $2 \leqslant 2 j<l$.

For $l$ odd $k\left(\lambda_{l}\right)=(l+1) / 2$.

For $l$ even $k\left(\lambda_{l-1}\right)=l$, and $k\left(\lambda_{l}\right)=l / 2$.

Also, all $\lambda_{j}^{*}=\lambda_{j}$.

$C_{i}: k\left(\lambda_{j}\right)=j$ for $1 \leqslant j \leqslant l$.

Also, all $\lambda_{j}^{*}=\lambda_{j}$.

$D_{l}: \quad k\left(\lambda_{2 j}\right)=k\left(\lambda_{2 j-1}\right)=2 j$ if $2 \leqslant 2 j \leqslant l-1$.

For $l$ even $k\left(\lambda_{l-1}\right)=k\left(\lambda_{l}\right)=l$.

For $l$ odd $k\left(\lambda_{l-2}\right)=l-1$,

and $k\left(\lambda_{l-1}\right)=k\left(\lambda_{l}\right)=(l-1) / 2$.

Also, if $l$ is odd $\lambda_{l}^{*}=\lambda_{l-1}$.

Otherwise $\lambda_{j}^{*}=\lambda_{j}$.

$G_{2}:\{2,2\} ; \lambda_{j}^{*}=\lambda_{j}$.

$F_{4}: \quad\{2,6,4,2\} ; \lambda_{j}^{*}=\lambda_{j}$.

$E_{6}:\{2,2,4,6,4,2\} ; \lambda_{1}^{*}=\lambda_{6}, \lambda_{3}^{*}=\lambda_{5}$,

$\lambda_{2}^{*}=\lambda_{2}, \lambda_{4}^{*}=\lambda_{4}$.

$E_{7}:\{2,5,6,8,7,4,3\} ; \lambda_{j}^{*}=\lambda_{j}$.

$E_{8}:\{4,8,10,14,12,8,6,2\} ; \lambda_{j}^{*}=\lambda_{j}$. 
ADDED IN PROOF. R. Palais pointed out to us that many properties of polar representations were already discussed in "A class of variationally complete representations” by L. Conlon, J. Differential Geom. 7 (1972), 149-160. The major point of our paper however is their classification, and its consequence Proposition 6.

\section{REFERENCES}

1. J. Dadok and V. Kac, Polar representations, J. Algebra (to appear).

2. J. Dadok and F. R. Harvey, Calibrations in $\mathbf{R}^{6}$, Duke Math. J. 50 (1983), 1231-1243.

3. S. Helgason, Differential geometry and symmetric spaces, Academic Press, New York, 1981.

4. __ Analysis on Lie groups and homogeneous spaces, CBMS Regional Conf. Ser. in Math., no. 14, Amer. Math. Soc., Providence, R. I., 1972.

5. J. E. Humphreys, Introduction to Lie algebras and representation theory, Springer-Verlag, New York, 1972.

6. G. A. Hunt, $A$ theorem of Élie Cartan, Proc. Amer. Math. Soc. 7 (1956), 307-308.

DEPARTMENT OF MATHEMATICS, INDIANA UNIVERSITY, BloOMINGTON, INDIANA 47405 